\title{
Positional discriminability in linear orderings
}

\author{
FREDRIC D. WOOCHER \\ Stanford University, Stanford, California 94305 \\ ARNOLD L. GLASS \\ Rutgers University, New Brunswick, New Jersey 08903 \\ and \\ KEITH J. HOLYOAK \\ University of Michigan, Ann Arbor, Michigan 48104
}

\begin{abstract}
Subjects were taught two eight-term linear orders of the form "A taller than B taller than C...." They were then asked to choose the "taller" term in all possible pairwise combinations within each series, and reaction time was measured for each pair. In addition, subjects performed a further task in which they judged whether or not two terms were adjacent in the ordering. In subsequent sessions, subjects were told that the "shortest" term on one list was taller than the "tallest" term on the other, so that the two lists were merged into a single 16-term series. They were then required to choose the "taller" term for both within-groups and between-groups pairs. Subjects did not appear to use the initial groupings in performing this task, even when given training on differential categorical codes ("tall" vs. "short") for the two sublists. Rather, subjects in all tasks appeared to represent the items as ordered positions along an internal array, so that comparison times depended largely on the differential discriminability of the item positions. In each task decisions were made more quickly if the terms being compared were near the ends of the ordering, rather than near the middle.
\end{abstract}

The problem of how information about serial order is stored in memory has concerned psychologists from Ebbinghaus (1885/1964) to the present (for an excellent historical review, see Crowder, 1976, Chapter 12). This classic problem has recently been investigated in a relatively new reaction time paradigm, in which subjects answer questions about an arbitrary ordering of stimuli along a dimension. A typical procedure is to first train the subjects on adjacent pairs of stimuli (e.g., Henry taller than Pete, Pete taller than Bill, Bill taller than Steve, etc.), and then ask questions about all possible pairs (e.g., Pete taller than Steve?). This paradigm began with the study of "three-term series problems" (Burt, 1919; Clark, 1969; DeSoto, London, \& Handel, 1965; Huttenlocher, 1968). More recent investigators have used larger series and emphasized different experimental phenomena; however, the primary emphasis of the earlier work, the process by which people make transitive inferences, remains a major concern (Trabasso, 1975).

This paper is based on a portion of a dissertation submitted to Stanford University by F. Woocher. We thank John Anderson, Gordon Bower, Herbert Clark, Keith Patterson, and Edward Smith for their advice and criticism. We are grateful to Debbie Shelby and Diane Christopherson for their work in testing subjects and analyzing the data. Portions of this study were supported by NIMH Grants RO3MH28225 to A. Glass, MH-13950-08 to G. Bower, and 20021 to $\mathrm{H}$. Clark; and by NSF Grant BNS77-01211 and a Rackham grant to K. Holyoak. Send reprint requests to K. Holyoak, Human Performance Center, 330 Packard Road, Ann Arbor, Michigan 48104.
A major finding in the recent work on linear orderings is that during the testing phase, subjects can compare nonadjacent pairs more quickly than they can compare adjacent pairs (Potts, 1972, 1974; Trabasso \& Riley, 1975; Trabasso, Riley, \& Wilson, 1975). The advantage of the nonadjacent or "remote" pairs is particularly interesting in that these pairs are not presented during training. Subjects are actually faster to compare items never previously paired (e.g., B > D) than to compare items that were explicitly learned together (e.g., B $>C$ ). It seems that subjects do not simply store the adjacent pairs during training and then use them to draw transitive inferences at the time of testing (as an "associative chaining" model might suggest). Rather, people appear to form a unified representation of the entire ordering during learning, so that comparison latencies then depend on emergent properties of the entire ordering rather than on the particular pairs from which the ordering was induced.

These linear order studies depart from the traditional serial order memory paradigm, the serial anticipation method, in several ways. The main dependent measure in the linear order work is usually reaction time (RT), rather than percent correct or trials to criterion. The task involves evaluating the relation between two stimuli, rather than using one stimulus as a cue to recall the next. However, there is also evidence that suggests the linear ordering paradigm taps some of the same mental processes as are involved in other serial learning tasks. In particular, studies of linear order judgments 
have produced a version of the most ubiquitous result in serial order research: the bow-shaped serial position curve. For example, consider a series of six terms, A through $F$. The time required to evaluate the five possible adjacent pairs is not uniform; rather, the end pairs $\mathrm{A}>\mathrm{B}$ and $\mathrm{E}>\mathrm{F}$ are compared relatively quickly, while the middle pair, $C>D$, is compared relatively slowly (Potts, 1974). In general, any pair involving an end item ( $A$ or $F$ ) is evaluated especially quickly (the "end anchor" effect). Like the classic serial position curve derived from learning studies, the bowed RT curve is asymmetrical. The direction of the asymmetry seems to depend on the question. For example, if A is the largest term and $F$ the smallest, pairs close to the A term anchor will be evaluated most quickly for the question, "Which is larger?", while pairs close to the F term anchor will be evaluated most quickly for "Which is smaller?" (McKinley, 1975; Trabasso \& Riley, 1975).

Theoretical links between serial learning and linear order tasks have also been proposed. A major theoretical account of serial learning is based on the "positional discriminability hypothesis" (Crowder, 1976). This view assumes that ordered stimuli are mapped onto an abstract or spatial internalized array, and that central positions in a linear array are less discriminable than end positions. Explicit versions of the positional discriminability hypothesis have been developed by Bower (1971) and Murdoch (1960). This view of serial learning can be extended very naturally to the linear order paradigm (DeSoto et al., 1965; Huttenlocher, 1968; Potts, 1974). Trabasso and Riley (1975) have explicitly applied Murdoch's (1960) discriminability model to the linear order paradigm. The positional discriminability model can account for the major differences found among comparison RTs: The farther apart two positions fall in the ordering, the more discriminable they will be; but with ordinal separation held constant, positions near the ends of the series will be more discriminable than positions near the center.

The aim of the present study was to obtain a wider range of data concerning the retrieval of information from linear orders, in order to further evaluate the positional discriminability hypothesis and competing proposals. As well as attempting to replicate the basic linear order results, two new experimental procedures were introduced.

\section{Merging Two Linear Orders}

Suppose subjects are first taught an ordering of two eight-term series (e.g., $\mathrm{A}>\mathrm{B}>\mathrm{C}>\mathrm{D}>\mathrm{E}>\mathrm{F}>\mathrm{G}>\mathrm{H}$ and $\mathrm{I}>\mathrm{J}>\mathrm{K}>\mathrm{L}>\mathrm{M}>\mathrm{N}>\mathrm{O}>\mathrm{P}$ ). If they are later told one additional pairwise relation, $\mathrm{H}>\mathrm{I}$, the two series can be merged into a single 16 -term series. We can then compare reaction times (RTs) to make within- groups comparisons (e.g., $\mathrm{C}>\mathrm{F}$ or $\mathrm{J}>\mathrm{M}$ ) vs. betweengroups comparisons (e.g., $G>J$ ). Which type of comparison will be made more quickly? This procedure might be expected to induce a hierarchical grouping of items, so that the list would be represented as two sublists, one of "large" items and the other of "small" items. Then if any "large" item is paired with any "small" item (i.e., a between-groups comparison), the categorical information could be used to produce a rapid decision. Such a grouping model could be applied to the standard linear order paradigm as well, if it is assumed that subjects naturally divide any ordering into subsequences. A grouping model could account for the relative ease of remote pairs, assuming that between-groups comparisons are fast relative to withingroups comparisons: The farther apart two items are in the ordering, the more likely they will be coded into different groups. One problem with such a model is that it would seem to predict a step function for RTs; that is, RTs should be very short for pairs of items that cross a structural break and very long for those that do not. However, with the additional assumption that the groupings vary across subjects, or even within subjects at different times during the testing procedure, a grouping model can predict a continuous decrease in RT as ordinal separation is increased.

The plausibility of grouping models is increased by the related research of Restle (1973), which suggests that hierarchical organization governs the learning of serial patterns. The concept of a major structural break in a continuum can also be invoked to explain some of the "symbolic distance effects" obtained with natural orderings. For example, Moyer (1973) found that subjects could choose the larger of a pair of animals very different in size (e.g., elephant-ant) more quickly than they could choose the larger of a pair close in size (e.g., elephant-horse). It is plausible that people have some animals classified as basically "large" (such as horses and elephants) and others as basically "small" (such as ants), and can use such categorical information to evaluate relative size. Also, Banks, Fujii, and Kayra-Stuart (1976) have proposed a model to account for the distance effects found with digits (Moyer \& Landauer, 1967) that invokes the notion of a major encoding break along the number continuum. Holyoak and Walker (1976) report a study on the representation of magnitude information in semantic orderings (e.g., torrid, hot, warm, cool, cold, frigid), in which they discuss a possible categorical model of how such terms are coded (see also, Kosslyn, Murphy, Bemesderfer, \& Feinstein, 1977).

It is possible, however, that subjects could represent two combined orderings quite differently; they might actually merge them into a single extended linear series. The positional discriminability hypothesis would then predict a pattern of results comparable to that obtained with the shorter lists: fast RTs for pairs near the end 
terms (now A and P), and slow RTs for pairs near the center of the entire ordering (near $\mathrm{G}, \mathrm{H}, \mathrm{I}$, or $\mathrm{J}$ ). The present study was designed to evaluate these alternative outcomes of the list-merger manipulation.

\section{Adjacency Judgments}

Previous linear order studies have always involved "greater" or "lesser" judgments about pairs of stimuli. However, other questions about the relations between ordered stimuli could be investigated. The present study included judgments of whether two terms drawn from an eight-term series were or were not adjacent in the ordering. If subjects can use the internalized ordering apparently accessed in the standard linear order paradigm to make adjacency judgments as well, then the RT patterns obtained in the two tasks should exhibit certain similarities. In particular, the positional discriminability hypothesis would predict a bow-shaped serial position curve, with adjacency judgments made relatively slowly for pairs near the middle of the series.

\section{METHOD}

The subjects were eight Stanford University undergraduates who were paid $\$ 8$ to participate in a series of three experimental sessions, spaced 1 week apart and each lasting approximately $1 \mathrm{~h}$.

Materials consisted of two eight-term ordered lists. Each ordering consisted of eight two-syllable occupation names taken from the Battig and Montague (1969) norms, roughly equated for length in letters and production frequency. The relation used in forming the ordering was always the linguistically unmarked "taller than." Each ordering was read to the subject by presenting the seven pairwise comparisons that comprised the ordering (e.g., "I am now going to read you a list of eight people who are arranged in order from tallest to shortest. The butcher is taller than the baker. The baker is taller than the grocer..."). Each subject received two different lists in this manner. The order of the terms within each list was reversed for half of the subjects. The lists were read to each subject at least 1 day before the first experimental session. The subject was told to copy them down, and to learn the lists (in serial order) the night before the first session.

\section{Procedure}

Session 1: Eight-term orders. A set of 56 test cards, consisting of all possible pairwise comparisons of the eight words (both orders of each pair) was constructed for each list. On each card the two words were typed side by side on one line. separated by a .5 -in. space. The two lists were tested in blocks, and the order of testing was counterbalanced across subjects. At the beginning of each test block (as well as at the beginning of each later session), the subject was asked to repeat the list fluently and without error; if he succeeded on the first try, he was allowed to perform the RT task. Subjects were invariably successful in reciting the list. In the experimental task, the subject had to decide which of two tachistoscopically presented occupation names was the taller, and to respond by pressing the button corresponding to that word (i.e., the left button for the left word, and the right button for the right word). The subject initiated each test trial by pressing a button, which caused a fixation point to appear for $1 \mathrm{sec}$. The test pair then appeared and a Hunter timer was started. The timer stopped when the subject responded. The experimenter then recorded the response and reaction time (in milliseconds) and informed the subject if he had made an error. The subject then initiated the next trial. The subjects were instructed to respond to each test sentence as rapidly as possible but not to sacrifice accuracy. At the beginning of the experimental session, subjects were given 15 practice trials in which they judged the larger of digit pairs, in order to familiarize them with the apparatus and testing procedure.

Each subject received all 56 possible pairwise combinations for each linear ordering, for a total of 112 experimental trials. The order of presentation of the test pairs was the same for each subject, but was randomized with the constraint that no pair appeared twice (in opposite order, e.g., A-B and B-A) within five trials, and no particular item appeared in more than three consecutive trials.

Session 1: Adjacency judgments. Following completion of the above procedure, the final $20 \mathrm{~min}$ of the first session were used for adjacency judgments with the same two eight-term orders. Two test blocks were constructed, one for each of the two lists, and the order of presentation of the blocks was counterbalanced across subjects. In this task, the subject had to decide if two tachistoscopically presented occupation names were adjacent to each other in the linear ordering, and to respond by pressing one of two decision buttons. Assignment of "true" and "false" to the two buttons was counterbalanced across subjects. The trial sequence consisted of a randomized order of the 14 possible adjacent pairs (true), the 12 pairs of items two ordinal steps apart (false), and 2 pairs of items with a step size of three (false); the proportion of true and false responses was therefore equal over the course of the experiment. Prior to the actual test sequence, subjects were given 18 practice trials, using digits as stimuli, to familiarize them with the new testing procedure. In all other respects, the procedure was identical to that of the earlier task.

Session 2: Sixteen-term order. At the beginning of the second session, subjects were told that the shortest person on one of the lists was taller than the tallest on the other list. For one group of subjects, List 1 was assigned to be the taller list; for the other group (those who had received the original lists in reverse serial order), List 2 was assigned to be the taller list. In this way, two 16 -term orderings were derived, differing only in whether the same items were ordered from tallest to shortest or from shortest to tallest. A subset of 96 of the possible 240 pairs was used as test pairs, consisting of all possible combinations (in both possible orders) of items one, three, five, and seven step sizes apart. Of this total, a third were comparisons between the two halves of the ordering, and the remainder were drawn in equal numbers from within each half of the ordering. A set of 15 practice items, using other pairs not included in the test set, preceded the actual test trials The order of presentation was randomized with the constraint that no identical pairs could appear within five trials of each other, and no single item could appear in more than two consecutive trials. The actual testing procedure was identical to that employed previously.

Session 3: Sixteen-term order retested. In Session 3 all the pairs used in Session 2 were retested. after some subjects performed a new training task. Four subjects were randomly selected to receive additional training designed to make the division of the 16-term list into two subsequences more salient. These subjects began the session with a now task, in which the list items were presented individually on the tachistoscope and the subject had to press one of two buttons as quickly as possible to indicate whether the word was a member of the "tall" or the "short" half of the 16-term list. This training session lasted about $15 \mathrm{~min}$, and consisted of three repetitions of the 16 items, presented in random order. Latencies on this task were not recorded, but performance was virtually error free. The remaining four subjects did not perform this task In the second part of the session, all subjects were retested on the paired comparisons of the 16-word list. The materials and procedure were identical to those used in the preceding session. 
Table 1

Mean Reaction Times by Condition for Eight-Term Orders

\begin{tabular}{|c|c|c|c|c|c|c|c|c|}
\hline \multirow[b]{2}{*}{ Condition } & \multicolumn{7}{|c|}{ Step Size } & \multirow[b]{2}{*}{ Mean } \\
\hline & 1 & 2 & 3 & 4 & 5 & 6 & 7 & \\
\hline $\begin{array}{l}\text { No Anchors } \\
\text { One Anchor } \\
\text { Two Anchors }\end{array}$ & $\begin{array}{l}1618 \\
1118\end{array}$ & $\begin{array}{l}1459 \\
1296\end{array}$ & $\begin{array}{l}1412 \\
1272\end{array}$ & $\begin{array}{l}1374 \\
1304\end{array}$ & $\begin{array}{l}1160 \\
1081\end{array}$ & 1049 & 834 & $\begin{array}{r}1471 \\
1187 \\
834\end{array}$ \\
\hline Mean & 1475 & 1405 & 1356 & 1339 & 1107 & 1049 & 934 & 1327 \\
\hline
\end{tabular}

\section{RESULTS AND DISCUSSION}

\section{Eight-Term Orders}

The error rate for comparisons based on the eightterm orders was very low (1.4\%). Reaction times greater than $3,000 \mathrm{msec}$ were truncated to that value, and all analyses were performed on correct RTs only. Table 1 presents mean RTs broken down by ordinal difference between the two terms (step size) and number of end anchors in the pair (zero, one, or two). These means are averaged over the two lists and pair order (left-right vs. right-left). As in previous studies, RT decreased as the distance between the items increased from Step Size 1 to Step Size $7[F(1,90)=92.4, p<.001$, by a linear trend test]. This distance effect is clearly present even when only the terms without end anchors are considered $[F(4,60)=42.0, p<.001]$.

Table 1 also reveals that RT decreased as the number of end anchors in the pair increased from zero to two $[F(2,30)=213, p<.001]$. A linear distance effect was not obtained for pairs containing an end anchor $[F(1,75)=2.04, p>.05]$. Reaction time was faster for pairs containing the "tall" anchor $(987 \mathrm{msec})$ rather than the "short" anchor $(1,352 \mathrm{msec})$, presumably reflecting the usual bias in favor of the end congruent with the comparative used in the question (i.e., "taller") (McKinley, 1975; Trabasso \& Riley, 1975).

Figure 1 plots the mean RT for each pair as a function of step size and the larger term of the pair. This graphical display of the data clearly shows that serial position effects occurred within any given distance. For Step Size 1 (the adjacent pairs), the serial position curve resembles the classic bow shape. As the ordinal distance between the two terms increases, the curves begin to flatten and become increasingly linear, as if the final portions of the bow-shaped curve had been successively truncated. It is interesting to note that there is a wide range of reaction times within any given ordinal distance, and that even when the pairs including end terms are ignored, reaction times between pairs of different step sizes often overlap. For example, response latencies were faster to pairs B-C and C-D than to pairs D-F and E-G, even though the former are adjacent pairs and the latter are two steps apart. These results are quite consistent with those of previous investigators (McKinley, 1975; Trabasso \& Riley, 1975) and indicate that serial position effects are not due to the end anchors alone, but are more continuous. Both the distance and serial position effects obtained here are consistent with the positional discriminability hypothesis. ${ }^{1}$

\section{Sixteen-Term Order}

The overall mean RT was much longer for the first day of testing on the 16-term list $(1,935 \mathrm{msec})$ than for the separate 8-term lists tested previously $(1,327 \mathrm{msec})$. The error rate on the 16 -term list was still quite low (3.6\%), however, and was virtually identical for between-groups and within-groups comparisons.

Mean correct RTs for the various conditions are presented in Table 2. The most important finding is that, contrary to the expectation of a grouping model, pairs containing items from different halves of the list were evaluated more slowly than pairs containing items from the same half. This trend was maintained even when within-groups comparisons containing end

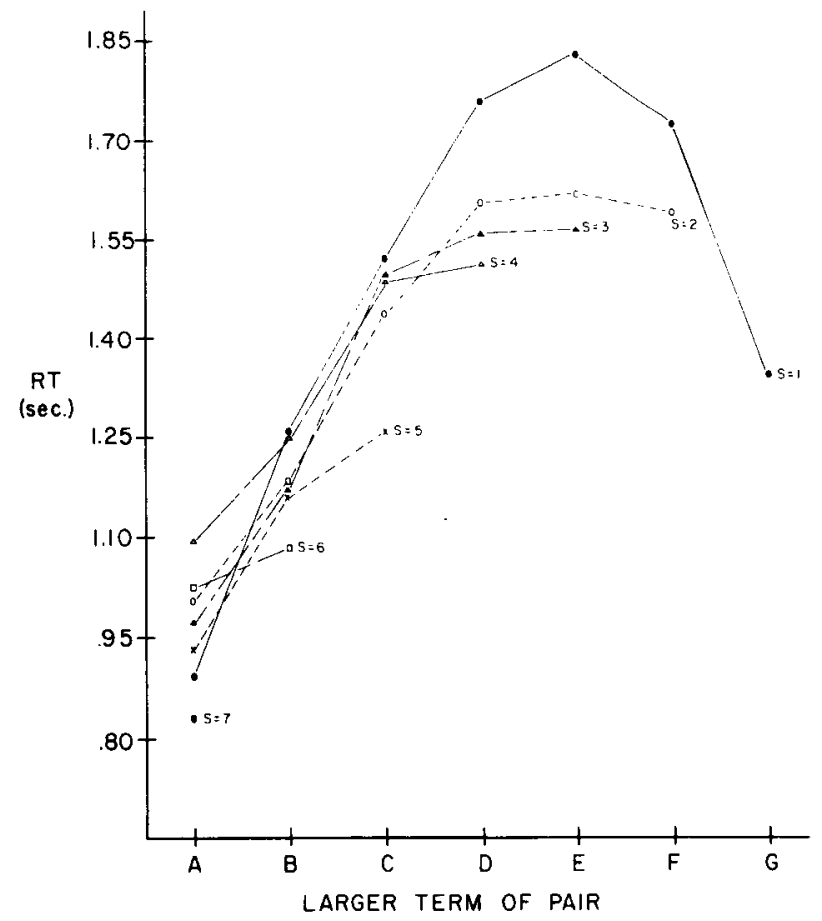

Figure 1. Mean RTs for eight-term ordering as a function of step size (S) and serial position (A is the "tallest" term). 
Table 2

Mean Reaction Times by Condition for Sixteen-Term Order

\begin{tabular}{|c|c|c|c|c|c|}
\hline \multirow[b]{2}{*}{ Condition } & \multicolumn{4}{|c|}{ Step Size } & \multirow[b]{2}{*}{ Mean } \\
\hline & 1 & 3 & 5 & 7 & \\
\hline No Anchors (overall) & 2086 & 2069 & 1983 & 1853 & 2017 \\
\hline One Anchor (overall) & 1367 & 1529 & 1468 & 1742 & 1526 \\
\hline Within Groups (all pairs) & 1993 & 1885 & 1659 & 1742 & 1881 \\
\hline Within Groups (no anchors) & 2097 & 1974 & 1754 & & 1998 \\
\hline Between Groups & 1941 & 2324 & 2166 & 1853 & 2044 \\
\hline Mean & 1990 & 1986 & 1889 & 1828 & 1935 \\
\hline
\end{tabular}

anchors were excluded, although the difference was not significant $[F(1,21)=2.11, p<.10]$. In addition to this major result, strong end anchor effects were obtained (when only the $\mathrm{A}$ and $\mathrm{P}$ terms were considered end anchors). Reaction times to pairs involving either the first or last term of the 16-term ordering were consistently faster than those involving no end items $[F(1,21)=113, p<.001]$. As with the eight-term orders, this effect was mainly due to pairs including the "tallest" term (mean $\mathrm{RT}=1,139 \mathrm{msec}$ ) rather than the "shortest" term (mean RT $=1,914 \mathrm{msec}$ ). A monotonic decline in overall RT with increasing distance was also obtained, although this trend was just barely significant $[F(1,21)=4.38, p<.05]$. However, overall distance was confounded with the relative number of between- vs. within-groups comparisons: The farther apart two items are, the more likely that they fall in different halves of the ordering. When between-groups and within-groups comparisons are examined separately, Table 2 reveals that clear distance effects were obtained for both types of comparisons.

Figure 2 plots the mean RTs for each test pair, averaged over pair order. While the data contain quite a bit of noise, the obtained serial position effects bear a striking resemblance to those obtained with the separate eight-term orders (Figure 1). Some of the apparent noise was due to a few rather inconsistent dips in RT for pairs containing the "middle" pairs H and I (i.e., pairs that were end anchors for the eight-term orders). But apart from these possible minor reflections of the grouping structure, the data suggest that subjects in fact merged the two lists into a single "mental array." In general, RT in this task appears to reflect the relative positional discriminability of terms within the entire list, with little or no residual effect of the subsequences from which the list was derived.

It might be supposed that the between-groups comparisons did not show an advantage because the items in each eight-term series had not been explicitly paired with uniform "size codes" during the initial session. Accordingly, in the final session, the 16-term series was retested immediately after half of the subjects received training in responding "tall" vs. "short" to items from the two half lists. The mean RTs for this second session with the full list are presented in Table 3 .
The error rates in this session were $3.4 \%$ for the control group (no pretraining) and $2.5 \%$ for the experimental group (given pretraining). Overall mean RT was substantially faster than in the first session with the 16-term series $(1,603$ vs. $1,935 \mathrm{msec})$. However, the pattern of results for both groups was essentially identical to that obtained in the preceding session. Both end anchor and distance effects (for pairs without $\mathrm{A}$ or $\mathrm{P}$ end terms) were again apparent. The pretraining procedure was entirely ineffective in producing any tendency for subjects to respond more quickly to between-groups than within-groups pairs; for both groups of subjects, the trend was again in the opposite direction. Figure 3 illustrates the serial position effects obtained for each ordinal distance (for the two groups combined). The general pattern is virtually identical to that found in the two earlier sessions (Figures 1 and 2): bow-shaped serial position curves, tending to flatten

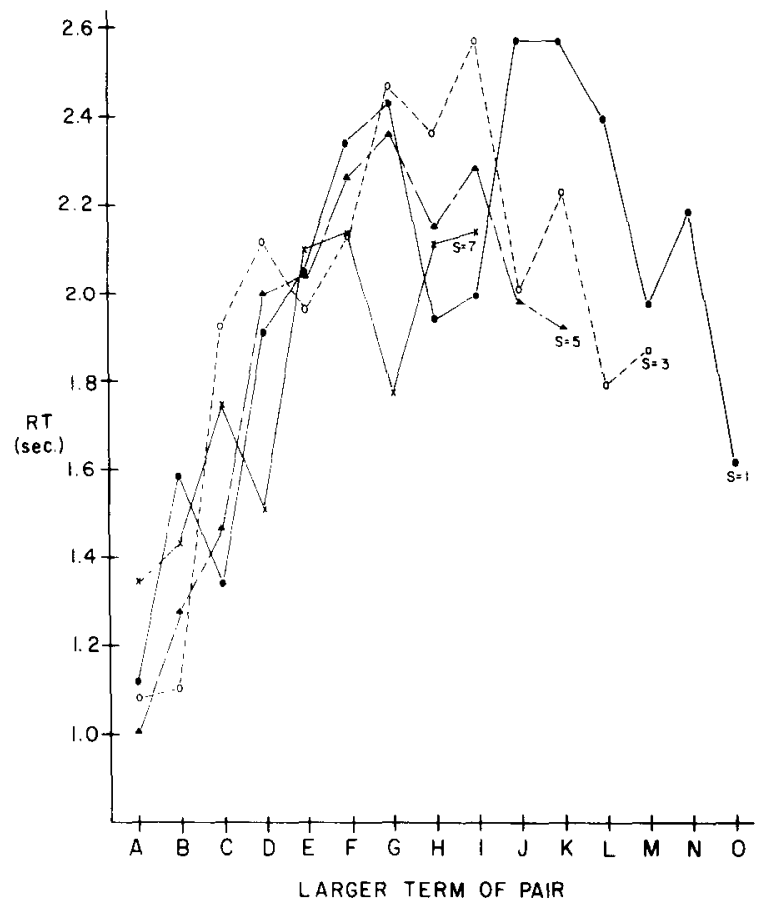

Figure 2. Mean RTs for 16-term ordering (first testing) as a function of step size (S) and serial position (A is the "tallest" term). 
Table 3

Mean Reaction Times by Condition for Retesting of Sixteen-Term Order

\begin{tabular}{|c|c|c|c|c|c|c|c|c|c|c|}
\hline \multirow[b]{3}{*}{ Condition } & \multicolumn{5}{|c|}{ Experimental Group (Pretraining) } & \multicolumn{5}{|c|}{ Control Group (No Pretraining) } \\
\hline & \multicolumn{4}{|c|}{ Step Size } & \multirow[b]{2}{*}{ Mean } & \multicolumn{4}{|c|}{ Step Size } & \multirow[b]{2}{*}{ Mean } \\
\hline & 1 & 3 & 5 & 7 & & 1 & 3 & 5 & 7 & \\
\hline No Anchors (overall) & 1734 & 1690 & 1704 & 1606 & 1695 & 1711 & 1723 & 1610 & 1465 & 1648 \\
\hline One Anchor (overall) & 1247 & 1312 & 1528 & 1529 & 1404 & 1049 & 931 & 1078 & 1396 & 1114 \\
\hline Within Groups (all pairs) & 1664 & 1545 & 1498 & 1529 & 1587 & 1591 & 1423 & 1411 & 1396 & 1492 \\
\hline Within Groups (no anchors) & 1734 & 1603 & 1482 & & 1648 & 1681 & 1546 & 1577 & & 1619 \\
\hline Between Groups & 1734 & 1921 & 1881 & 1606 & 1759 & 2069 & 2195 & 1637 & 1465 & 1693 \\
\hline Mean & 1669 & 1632 & 1672 & 1589 & 1647 & 1623 & 1601 & 1514 & 1450 & 1559 \\
\hline
\end{tabular}

as step size increases. The results are again consistent with the view that RT is primarily determined by the positional discriminability of items within the entire 16-term ordering.

This conclusion derives further support from the results of multiple-regression analyses performed (separately for the two sessions) on the mean RTs for the 16-term ordering. In each case four "distance from end anchor" variables accounted for most of the systematic variance (about $45 \%$ of the total variance). These were the minimal distance from an end term (A or P) and the distance from the "tallest" term (A); both of these variables were significant for both the "taller" and the "shorter" pair members. These variables are essentially the same as those that predicted RTs for the eight-term orders. The distance between the two terms in a pair accounted for a small but significant amount of residual variance. However, the factor of within-groups vs. between-groups comparisons was not significant after the variance due to distance and end anchor variables was removed. For the test items used in the present study, the items in between-groups pairs were relatively far apart in the ordering, but also relatively far from an end anchor (A or P). Since the latter variable had a greater influence on RT, decisions about between-groups pairs tended to be relatively slow. However, the multiple-regression results indicate that list position factors were the critical determinants of RT, and the between-groups vs. within-groups factor had no direct influence on the results.

\section{Adjacency Judgments}

The error rate on the adjacency task (which followed the eight-term series judgments on Session 1) was $5.8 \%$. Mean RTs over 4,000 msec were truncated to that value. Figure 4 displays mean RT to respond "true" for each adjacent pair, ordered from "tallest" to "shortest." The curves are plotted separately for both presentation orders (e.g., A-B vs. B-A), as well as collapsing over order; the data for the two lists are combined. The asymmetrical bow-shaped curve emerges once again in this new type of judgment. In fact, the serial position effects obtained for adjacency judgments are remarkably similar to the pattern obtained for "taller" judgments about the same pairs (Figure 1). This pattern is consistent with the hypothesis that subjects used an internal array to perform this task as well, and that differences in RT again reflect differences in the discriminability of item positions along the array.

Figure 4 also reveals that RT was faster overall when pairs were presented with the "taller" term on the left ("correct" order) rather than on the right ("reverse" order) $[F(1,56)=41.8, p<.01]$. However, the advantage of the correct order disappeared for pairs near the "shorter" end of the list (E-F, F-G, G-H) $[\mathrm{F}(7,56)=9.1$, $p<.011$. These order effects suggest that something more than positional discriminability (which should not vary with order) affects RT in this task. One

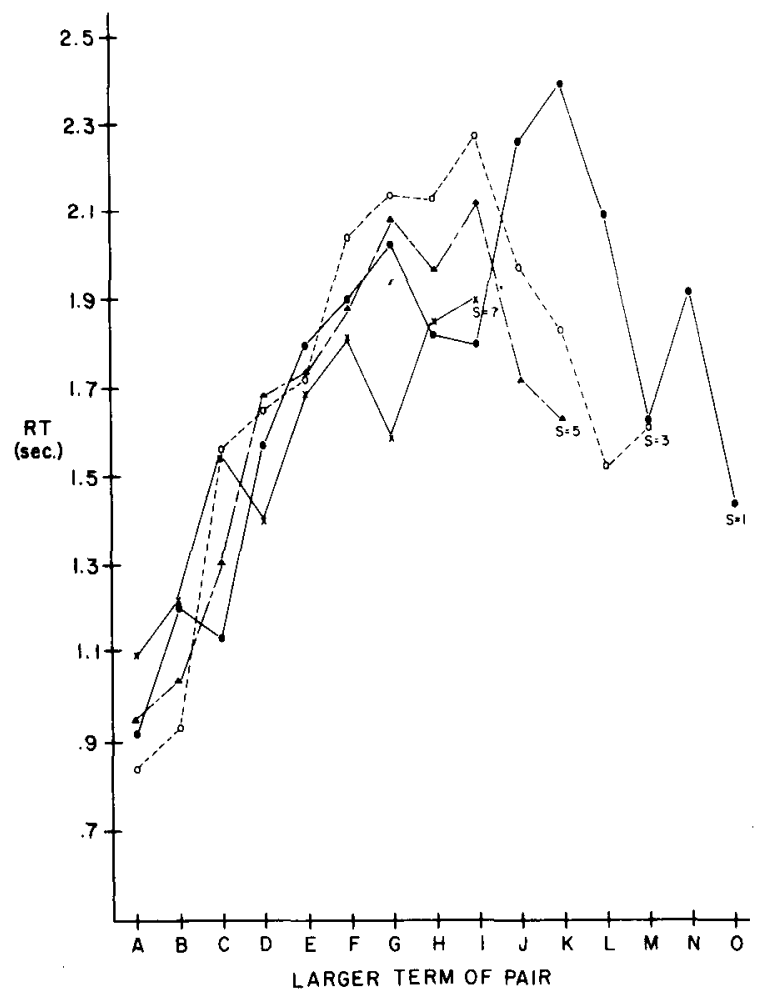

Figure 3. Mean RTs for 16-term ordering (second testing) as a function of step size (S) and serial position ( $A$ is the "tallest" term). 


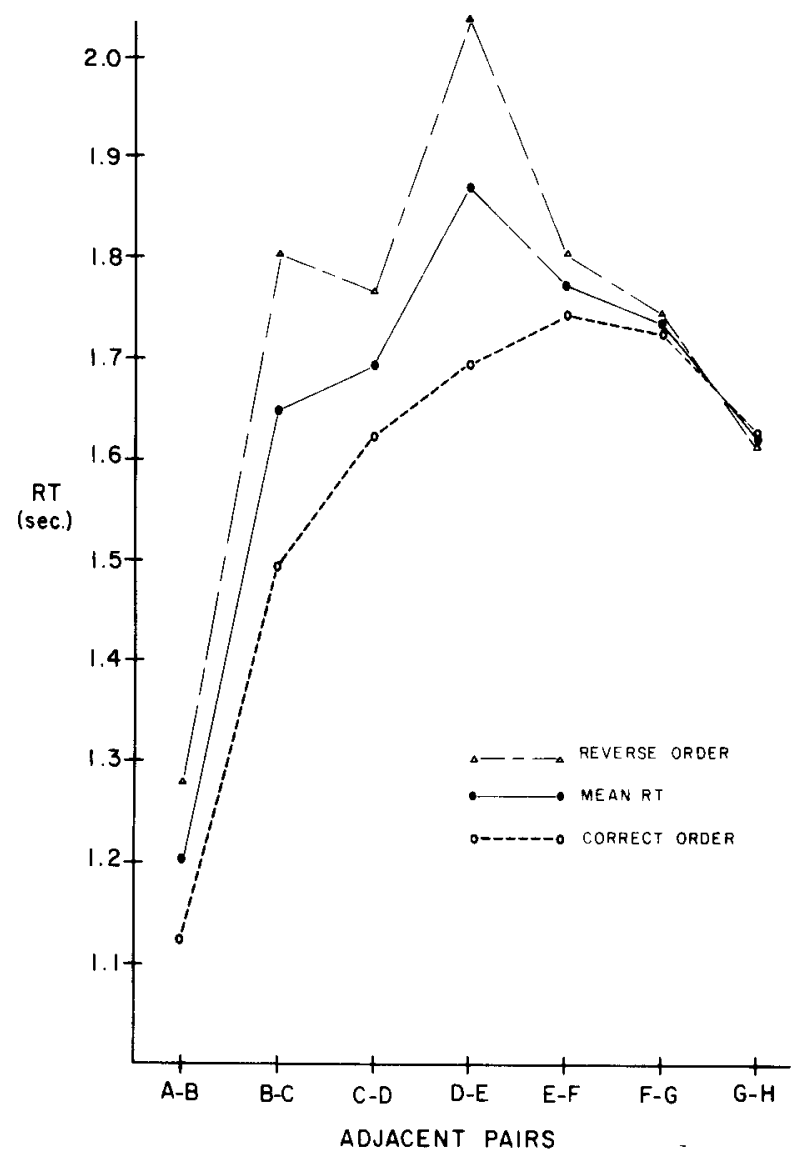

Figure 4. Mean RTs for adjacency judgments ("true" cases only) as a function of serial position and order of the pair.

possibility is that adjacency judgments involve a search component; the left item (which is probably read first) is first located in the array, initiating a "scan" from that array position to locate and identify adjacent items. It seems plausible that such a scan may have a directional bias. Since the list was to be learned in the order "tallest" to "shortest," the scan would generally move from the "tall" items in toward the center of the list; however, the "short" items may have tended to be acquired "from the end in" (Trabasso et al., 1975), rendering them relatively neutral with respect to direction of scan. Such directional biases may have produced the obtained interaction between presentation order and serial position. A scanning model of the above sort might generally account for the bow-shaped serial position curves found in linear order studies, and thus provide a mechanism to explain the differential availability of positions in an array. Woocher (1976) has formulated a mathematical version of an endsinward search model and applied it successfully to the data from 8-term and 16-term orderings.

The mean RTs to respond "false" to the nonadjacent pairs are presented in Table 4. These also show an asymmetrical bow-shaped serial position function, although it is less pronounced than that obtained with the adjacent pairs. Since all but one of the pairs had a step size of two between the items, no strong conclusion can be reached about the effect of the distance between the items on time to decide a pair is not adjacent. Nevertheless, the fact that the one pair with Step Size 3, C.F, tended to be evaluated relatively quickly is at least suggestive. If speed to reject a pair as nonadjacent in fact increases with the distance between the items, this would provide further evidence that discriminability along an internal array also influences adjacency judgments.

\section{GENERAL DISCUSSION}

The results of the present study are consistent with the hypothesis that serial order information is coded in memory as an internalized array. The ease of retrieving information from the array is largely governed by the relative discriminability of the relevant item positions, with end positions being more discriminable than central ones. This conclusion appears to hold not only for decisions about which of two items is taller, but also for decisions about whether the two items are adjacent in the array. The latter result illustrates the general usefulness of examining different but related judgment processes, in order to find converging evidence for a single underlying knowledge structure. Our results provide a broader empirical base for models of serial order that incorporate the positional discriminability hypothesis (Bower, 1971; Murdoch, 1960; Trabasso \& Riley, 1975).

Perhaps the most surprising result of the present study is the failure to find any clear evidence that experimentally imposed groupings affect the time to retrieve information from a linear ordering. When two 8 -term series were combined in a single 16-term list, the existence of a clear break in the ordering did not facilitate comparisons of items that straddled the division. Rather, subjects appeared to treat the new list as a unified linear array, so that RTs were largely

Table 4

Mean Reaction Times for False Pairs in Adjacency Task

\begin{tabular}{|c|c|c|c|c|c|c|c|c|}
\hline \multirow{2}{*}{$\begin{array}{l}\text { Test } \\
\text { Pair } \\
\end{array}$} & \multicolumn{7}{|c|}{ Step Size 2} & \multirow{2}{*}{$\frac{\text { Step Size } 3}{\text { C-F }}$} \\
\hline & $A-C$ & B-D & $C-E$ & $D-F$ & $E-G$ & $\mathrm{~F}-\mathrm{H}$ & Mean & \\
\hline $\begin{array}{l}\text { Correct Order } \\
\text { Reverse Order }\end{array}$ & $\begin{array}{l}1557 \\
2115\end{array}$ & $\begin{array}{l}1820 \\
2131\end{array}$ & $\begin{array}{l}1938 \\
2155\end{array}$ & $\begin{array}{l}2027 \\
2016\end{array}$ & $\begin{array}{l}1944 \\
2167\end{array}$ & $\begin{array}{l}2017 \\
2088\end{array}$ & $\begin{array}{l}1884 \\
2112\end{array}$ & $\begin{array}{l}1760 \\
2017\end{array}$ \\
\hline Mean & 1836 & 1976 & 2047 & 2022 & 2056 & 2052 & 1998 & 1889 \\
\hline
\end{tabular}


determined by the positional discriminability of the items within the entire 16-item list.

The present results certainly do not conclusively indicate that between-groups comparisons are not facilitated in some way. In the present study, subjects received a session of practice on the within-groups comparisons before the trials on the 16-term series began; and even in the last two sessions, between-groups pairs occurred half as frequently as within-groups pairs. Nevertheless, it seems surprising that the relative difficulty of the between-groups pairs did not diminish at all in the final session. But in any case, it would be a mistake to interpret the present results as a demonstration that internal groupings within an ordering never influence performance. A variety of related research (Martin, 1974; Martin \& Noreen, 1974; Restle, 1973) indicates that the internal grouping structure of a serial order can play an important role in tasks other than comparative judgment. In the present study, the two eight-term lists were not given differential codes (e.g., tall vs. short) during initial learning. Although half the subjects were given training on such codes before the final set of trials on the 16-item list, without producing any grouping effect, one could fairly argue that this training was "too little and too late." Indeed, Kosslyn et al. (1977) have demonstrated some effects of induced groupings on comparative judgment latencies, but only when subjects were first trained on the categorical codes to a very high criterion of overlearning. The present findings are an important positive demonstration, however, as they suggest that subjects can map two initially separate orderings onto a unified internal array. Even though the subjects apparently did not use the grouping structure, the derived ordering allowed them to immediately proceed to make any of the possible pairwise comparisons with a high degree of accuracy.

It might be argued that the much longer latencies obtained with the 16-term than with the 8-term orders (a difference of about $600 \mathrm{msec}$ overall) indicate a qualitative difference in processing. However, note that longer orders necessarily have items in the middle of the list that are relatively far from the ends. According to the positional discriminability hypothesis, the total amount of interitem interference will increase with list length, and the bulk of the effect will be found with pairs involving central items. In terms of an endsinward scanning model (Woocher, 1976), a longer search will be required to access the central items in a relatively long list. Accordingly, relative serial position effects should be magnified as list length increases. A comparison of Figures 2 and 3 with Figure 1 supports this prediction. Indeed, by the final session with the 16-term ordering (Figure 3), RTs for pairs containing end terms are evaluated about as quickly as they were in the 8-term orderings (Figure 1); pairs drawn from the center of the ordering, however, are still evaluated about $600 \mathrm{msec}$ slower than the slowest pairs in the 8-term series. If similar processes in fact operate for both short lists and ones as large as the 16-term ordering used in the present study, this result will be important for models of linear orderings. For example, the present results make it appear very unlikely that the phenomena obtained in linear order studies are solely due to shortterm memory processes.

An important task for future research is to clarify the relationship between the processes underlying judgments about order in an experimentally taught series, as in the present study, and judgments about the relative magnitude of terms ordered on some dimension (e.g., size) on the basis of semantic knowledge (Moyer, 1973). Many theorists have argued that some variety of spatial or analog knowledge is involved in both "artificial" and "natural" dimensional information. However, some significant differences in the patterns of comparison RTs for the two types of studies have been observed. While symbolic distance effects seem to be universally obtained, the bow-shaped position curves typical of judgments about experimentally taught orderings are generally absent when the judgments are based on real-world magnitude information. In particular, bow-shaped functions are generally not found in comparisons of digits (Banks et al., 1976; Buckley \& Gillman, 1974), animal sizes (Moyer, 1973), or terms from semantic orderings (Holyoak \& Walker, 1976). This suggests that, while magnitude information in semantic memory may be stored in an analog form, it is not represented in an internalized linear array in the same way as an experimentally acquired ordering appears to be.

While this and many other questions about the internal representation of order and magnitude information remain to be answered, the present results provide additional support for the view that subjects use a spatial position mechanism to integrate serial order information in memory.

\section{REFERENCES}

Banks, W. P., Fujir, M., \& Kayra-Stuart, F. Semantic congruity effects in comparative judgments of magnitudes of digits. Journal of Experimental Psychology: Human Perception and Performance, 1976, 2, 435-447.

Battic, W. F., \& Montague, W. E. Category norms for verbal items in 56 categories: A replication and extension of the Connecticut category norms. Joumal of Experimental Psychology Monograph, 1969, 80, 1-46.

Bower, G. H. Adaptation-level coding of stimuli and serial position effects. In M. H. Appley (Ed.), Adaptation-level theory. New York: Academic Press, 1971. Pp. 175-201.

Buckley, P. B., \& Gillman, C. B. Comparisons of digits and dot patterns. Journal of Experimental Psychology, 1974, 103, 1131-1136.

BuRT, C. The development of reasoning in school children. Journal of Experimental Pedogogy, 1919, 5. 68-77.

$\mathrm{C}_{\text {LARK, }} \mathrm{H}$. H. Linguistic processes in deductive reasoning. Psychological Review, 1969, 76, 387-404. 
Crowder, R. G. Principles of learning and memory. Hillsdale, N.J: Lawrence Erlbaum, 1976.

DeSoto, C. B., London, M., \& Handel, S. Social reasoning and spatial paralogic. Journal of Personality and Social Psychology, 1965, 2. 513-521.

Ebinghaus, H. E. Memory: A contribution to experimental psychology. New York: Dover. 1964. (Originally published 1885).

Holyoak, K. J., \& Walker. J. H. Subjective magnitude information in semantic orderings. Journal of Verbal Learning and Verbal Behavior, 1976, 15, 287-299.

Huttenlocher, J. Constructing spatial images: A strategy in reasoning. Psychological Review, 1968, 75, 550-560.

Kossly n, S. M., Murphy, G. L., Bemesderfer, M. E., \& Feinstein, K. J. Category and continuum in mental com. parisons. Journal of Experimental Psychology: General, 1977. 106, 341-375.

Martin, E. Serial learning: A multilevel access analysis. Memory \& Cognition, 1974, 2. 322-328.

Martin, E., \& Noreen, D. L. Serial learning: Identification of subjective subsequences. Cognitive Psychology, 1974, 6, 421-435.

MCKINLEY, J. E. Utilizing comparative information from memory: The symbolic distance effect. Unpublished Masters thesis, University of Massachusetts, 1975.

MOYER, R. S. Comparing objects in memory: Evidence suggesting an internal psychophysics. Perception \& Psychophysics, 1973, 13, 180-184.

Moyer, R. S., \& Landauer, T. K. Time required for judgments of numerical inequality. Nature (London), 1967. 215. 1519-1520.

MurDóch, B. B., JR. The distinctiveness of stimuli. Psychological Review, 1960, 67, 16-31.

PotTs, G. R. Information processing strategies used in the encoding of linear orderings. Journal of Verbal Learning and Verbal Behavior. 1972, 11, 727-740.
Potrs. G. R. Storing and retrieving information about ordered relationships. Journal of Experimental Psychology, 1974, 103, 431-439

Restle, F. Serial pattern learning: Higher order transitions. Journal of Experimental Psychology, 1973, 99, 61-69.

Trabasso, T. Representation, memory and reasoning: How do we make transitive inferences? In A. D. Pick (Ed.), Minnesota symposia on child psychology (Vol. 9). Minneapolis: University of Minnesota Press, 1975. Pp. 135-172.

Trabasso, T.. \& Riley, C. A. On the construction and use of representations involving linear order. In R. L. Solso (Ed.), Information processing and cognition: The Loyola symposium. Hillsdale, N.J: Lawrence Erlbaum, 1975.

Trabasso. T., Riley, C. A., \& Wilson, E. G. The representation of linear order and spatial strategies in order: A developmental study. In R. Falmagne (Ed.), Psychological studies of logic and development. Hillsdale, N.J: Lawrence Erlbaum, 1975.

WOOCHER, F. D. Storage and retrieval of information from artificial linear orderings. Unpublished $\mathrm{PhD}$ dissertation, Stanford University, 1976.

\section{NOTES}

1. The data were also submitted to multiple-regression analyses, which essentially confirmed the pattern apparent in Figure 1. The major variables predicting RT were the minimal distance from the items in a pair to one of the two end anchors, and in particular, the distance of the items from the "tall" end.

(Received for publication October 11, 1977; revision accepted October $26,1977$. 